\title{
Preferences for opt-in and opt-out enrollment and consent models in biobank research: a national survey of Veterans Administration patients
}

\author{
David Kaufman, PhD'1, Juli Bollinger, MS'1, Rachel Dvoskin, $\mathrm{PhD}^{1}$ and Joan Scott, MS, GCG ${ }^{2}$
}

\begin{abstract}
Purpose: In 2006, the Department of Veterans Affairs launched the Genomic Medicine Program with the goal of using genomic information to personalize and improve health care for veterans. A step toward this goal is the Million Veteran Program, which aims to enroll a million veterans in a longitudinal cohort study and establish a database with genomic, lifestyle, military-exposure, and health information. Before the launch of the Million Veteran Program, a survey of Department of Veterans Affairs patients was conducted to measure preferences for opt-in and opt-out models of enrollment and consent.

Methods: An online survey was conducted with a random sample of 451 veterans. The survey described the proposed Million Veteran Program database and asked respondents about the acceptability of opt-in and opt-out models of enrollment. The study examined differences in responses among demographic groups and relationships between beliefs about each model and willingness to participate.
\end{abstract}

Results: Most respondents were willing to participate under both opt-in (80\%) and opt-out (69\%) models. Nearly $80 \%$ said they would be comfortable providing access to residual clinical samples for research. At least half of respondents did not strongly favor one model over the other; of those who expressed a preference, significantly more people said they would participate in a study using opt-in methods. Stronger preferences for the opt-in approach were expressed among younger patients and Hispanic patients.

Conclusion: Support for the study and willingness to participate were high for both enrollment models. The use of an opt-out model could impede recruitment of certain demographic groups, including Hispanic patients and patients under the age of 55 years.

Genet Med 2012:14(9):787-794

Key Words: biobank; genetics; informed consent; opt-out; recruitment; research ethics

\section{INTRODUCTION}

Large, prospective cohort studies and biobanks are becoming standard research tools to study the roles of genes, environment, and lifestyle in health and disease., ${ }^{1,2}$ Analyzing genetic data (obtained from blood or tissue samples) together with clinical information (typically obtained from medical records) ${ }^{3-7}$ from large numbers of people makes it possible to begin to identify distinct genotypes and environmental factors that contribute to the development of common complex diseases. Studies involving hundreds of thousands of participants are needed to detect genetic variants and other factors that interact to confer modest increases in disease risk. The design of efficient methods to recruit and enroll large numbers of participants while protecting their rights as human subjects may be informed in part by the preferences and beliefs of the population being sampled.

Many biobanks seek informed consent at the time of enrollment, asking participants to actively opt in to the study before their information and samples are collected or used. Biobanks and large cohort studies that recruit participants from within health-care systems or public collections of samples and medical records have the option of using an opt-out method of enrollment. When patients' medical records in a health-care system can be linked to residual blood and tissue samples collected during clinical visits, a biobank may not need to collect additional data in order to conduct research on these patients. In such cases, patients can be informed of the study goals and given an opportunity to opt out if they do not want their samples and information to be used for research. Those who do not actively opt out of participating form the study population. ${ }^{6}$

One advantage of using an opt-out method is that it provides both researchers and participants a simple and convenient enrollment process. Opt-out methods may yield high enrollment and, in at least one study, have been shown to result in less biased ascertainment of cases. ${ }^{6}$ Possible disadvantages of this method include enrollment of people who do not wish to participate in the study but fail to opt out, the logistical burden of providing all potential participants the opportunity to opt out, and the creation of a study population that may be less committed to long-term participation given the passive nature of enrollment.

The Department of Veterans Affairs (VA) health-care system has a clinical population that is well suited to large-scale longitudinal research. A large proportion of the VA's 8.3 million patients visit a VA clinic annually, and many are seen repeatedly 
at the VA over long periods of time. In addition, the VA uses an electronic medical record system that aggregates patient information regardless of where in the VA system the patient is seen. ${ }^{7}$

In 2006, the VA established the Genomic Medicine Program (GMP) to increase the understanding of genetic and nongenetic causes of common diseases affecting both veteran and general populations. To support these goals, the VA has proposed and established the Million Veteran Program (MVP), a database of annotated clinical samples from consenting volunteer patients. ${ }^{8}$ In preparation for launch of the MVP, the VA undertook a public consultation with veterans to explore the VA patient population's support for, willingness to participate in, and preferences about the design and conduct of such research. ${ }^{9}$ In the initial consultation study, focus groups and a survey measured veterans' preferences and opinions about issues related to privacy, the return of research results, and informed consent. Eightythree percent of respondents said the database should definitely or probably be created; $71 \%$ said they would definitely or probably participate. Nearly half $(47 \%)$ of VA patients said they would prefer to give a one-time, broad consent for use of their samples and data in all research approved by the GMP; $43 \%$ said they would prefer to give separate consent for each research project undertaken using the database; and 10\% would prefer to select broad categories of acceptable research for the study at the outset. ${ }^{9}$

As a follow-up to that study, the survey described here was conducted to explore in more detail VA patient preferences regarding the enrollment process for the MVP. This national survey of veterans who use the VA health-care system compared the acceptability of opt-in and opt-out models for enrollment, explored how each model might operate in practice, and asked about the use of residual clinical samples for research. Differences between demographic groups with respect to these opinions were explored. The findings from this survey were presented to the GMP Advisory Committee with the intent of informing the design of an enrollment model that would be more likely to produce a representative sample of the VA patient population.

\section{Study population}

MATERIALS AND METHODS

Sample selection and online administration of the survey were managed by the survey research firm Knowledge Networks $(\mathrm{KN})$. The sample for this study was drawn from KN's master panel, which includes $\sim 43,000$ US residents over the age of 18 years selected using list-assisted random-digit dialing to produce a probability-based sample. To decrease bias resulting from a "technology gap," people selected for the KN panel who do not have Internet access are given free access and hardware by KN. To prevent panel members from becoming "professional respondents," the master panel is constantly refreshed and panel members are regularly replaced with new members.

To avoid collecting responses from veterans with prior knowledge of the GMP, KN panel members who had completed either the 2008 survey or a 2009 pilot study (total $N=496)$ were excluded. KN invited the remaining 3,789 nonactive military listed in their sample to participate. Because the GMP would be limited (initially) to VA patients, and because some veterans are not eligible for VA care and others choose not to use it, respondents were first screened to limit the sample to veterans who receive some health or psychological care from the VA. The online survey was then offered to those who have ever received or currently receive VA care.

\section{Survey content and administration}

A survey instrument was developed to measure attitudes, expectations, and concerns regarding the proposed VA biobank among veterans who use the VA health-care system. The 108item survey, with a reading grade level of 7.3 , was approved by the Johns Hopkins University institutional review board (application \#NA-00028193). Before the idea of the biobank was introduced in the survey, respondents were asked whether it is "a good idea to use leftover blood or tissue for research purposes" and what they "assume happens to leftover blood or tissue from a VA hospital visit." Respondents were then shown the same description of the database that was used in the 2008 survey $^{9}$ and asked about their support for the GMP, their attitudes about opt-in and opt-out methods of enrollment, and their preferences about how both models would operate. To examine whether the current survey sample was comparable to the 2008 sample, several questions about participants' attitudes toward the MVP were repeated in an identical format.

Survey participants were first presented the opt-out model of enrollment, in which VA patients would be informed about the GMP and told that residual samples (which are currently destroyed by the VA) would instead be automatically used, together with medical records, to populate the database unless a patient specifically opted out. After answering several questions about the opt-out model, participants were shown a description of an opt-in model, in which VA members would be invited to participate in the biobank and informed about what participation entailed; only after a person consented to participate would the VA obtain a biological sample and access their medical information (see Supplementary Data online for the exact description used). Participants were then asked several questions about the opt-in model.

Respondents were asked whether the database should be developed if it were carried out using an opt-out approach, and whether it should go forward using an opt-in approach. The order of these two questions was randomized. Definitions for key terms such as "opt-in" and "opt-out" were hyperlinked to the terms throughout the survey so that respondents could review them at any time. The survey concluded with several demographic questions related to participants' military service.

Before fielding the survey, a pilot was conducted between 2 and 4 May 2009, with 50 veterans randomly selected from the $\mathrm{KN}$ panel to evaluate the survey's length, logic, skip patterns, and wording. The survey was revised on the basis of responses to questions assessing comprehension of the two models, and 
suggestions from pilot participants were incorporated into the final instrument. Median time to complete the 131-item pilot survey was $25 \mathrm{~min}$. To reach the target survey length of $20 \mathrm{~min}$, the instrument was shortened to 108 items. The survey instrument included an explanation and schematic of the proposed database.

The survey was fielded online between 15 May and 4 June 2009. Potential participants were e-mailed an invitation to participate, and nonresponders received one e-mail reminder. Participants received $\$ 5$ for their time. After survey data were collected, prebanked information collected by KN on participant demographics was added to the data set.

\section{Data analysis}

Data were sorted and prepared for analysis using SPSS software. ${ }^{10}$ Data were analyzed using the SUDAAN software package, ${ }^{11}$ which employs Taylor-series linearization estimations of variances to correct for the survey-sampling scheme when judging hypothesis test results. Sampling strategy was corrected for using SUDAAN's “WOR” (without replacement) design option. Multiple logistic regressions were used to examine demographic factors and opinions associated with willingness to participate under the two models. Analyses that included the entire sample were weighted to 2005 VA demographic benchmarks ${ }^{12}$ for gender, race and ethnic background, age, education, and region of the United States, to reduce bias from sampling errors. Simple Bonferroni corrections were used to examine whether any demographic group was more likely to favor an opt-in or optout approach to enrollment-because each group's response to the opt-in option was compared with its response to the opt-out option. To assess changes in attitudes since the original 2008 survey and to examine whether the ascertainment strategy for the current survey (excluding any KN members who took the 2008 survey) biased the sample, we compared the responses to several questions asked in both 2008 and 2009, adjusting for demographic and service variables. Bonferroni corrections for multiple comparisons were also made when comparing the 2008 and 2009 survey samples.

\section{RESULTS}

Using previously collected data, KN identified and invited 3,789 panel members who listed themselves as nonactive military. Of these veterans, 3,026 responded and were asked to answer the initial screening question, yielding a response rate of $80 \%$. Of 3,026 screened respondents, 451 (15\%) qualified to take the survey because they received some health or psychological care from the VA and had not responded to the previous survey. The margin of error around opinion estimates based on a sample of 451 is $\pm 5.2 \%$, after correcting for sampling design. Table 1 compares distributions of demographic characteristics in the weighted sample of respondents to a study of VA demographics completed in 2005. ${ }^{12}$ The distributions of gender and race and ethnic background in this study sample were similar to 2005 VA demographics. As with our previous survey of VA patients from the KN panel, the survey sample had a somewhat higher
Table 1 Comparison of weighted distributions of demographics in the GPPC 2008 and 2009 survey samples and a 2005 Veterans Administration population survey

\begin{tabular}{|c|c|c|c|c|}
\hline \multirow{2}{*}{$\begin{array}{l}\text { Demographic group } \\
\text { Gender }\end{array}$} & \multicolumn{2}{|c|}{$\begin{array}{c}2009 \text { GPPC } \\
\text { sample, } \\
\text { weighted } \\
(n(\%))\end{array}$} & \multirow[t]{2}{*}{$\begin{array}{c}2008 \text { GPPC } \\
\text { sample, } \\
\text { weighted } \\
(\%)\end{array}$} & \multirow[t]{2}{*}{$\begin{array}{c}2005 \text { VA } \\
\text { demographic } \\
\text { data }^{a} \\
(\%)\end{array}$} \\
\hline & & & & \\
\hline Men & 422 & 94 & 93 & 93 \\
\hline Women & 29 & 6 & 7 & 7 \\
\hline \multicolumn{5}{|l|}{ Race } \\
\hline White non-Hispanic & 359 & 80 & 80 & 80 \\
\hline Black non-Hispanic & 48 & 10 & 10 & 10 \\
\hline Hispanic (all races) & 25 & 6 & 4 & 5 \\
\hline Other non-Hispanic & 19 & 4 & 6 & 5 \\
\hline \multicolumn{5}{|l|}{ Age (years) } \\
\hline $18-44$ & 94 & 21 & 14 & 14 \\
\hline $45-64$ & 199 & 44 & 42 & 41 \\
\hline$\geq 65$ & 158 & 35 & 44 & 45 \\
\hline
\end{tabular}

Education

$\begin{array}{lllll}0-12 \text { Years } & 169 & 37 & 39 & 41 \\ \text { Some college } & 172 & 38 & 37 & 36 \\ \text { Bachelor's degree } & 110 & 25 & 24 & 23\end{array}$

GPPC, Genetics and Public Policy Center; VA, Department of Veterans Affairs. aSource: Department of Veterans Affairs Office of the Assistant Deputy Under Secretary for Health Policy and Planning, 2006. 2005 Survey of Veteran Enrollees Health and Reliance Upon VA.

level of education and included a larger proportion of people between the ages of 45 and 64, as compared with the overall VA population (Table 1 ).

\section{The use of residual clinical samples in research}

Survey participants were first asked for their general opinions about the use of residual clinical samples for research. Nearly half (49\%) of respondents assumed that leftover blood or tissue from VA hospital visits is discarded, whereas $4 \%$ assumed they were already being stored or used for research. A total of $47 \%$ were unsure of what happens to these samples. A total of $77 \%$ agreed that it is "a good idea to use leftover blood or tissue for research purposes.” Black non-Hispanics (64\%) were less likely than white non-Hispanics (78\%), Hispanics (84\%), and nonHispanics of other races $(79 \%)$ to support the use of leftover samples for research $(P=0.03)$. Women were more likely than men to support the use of such samples, although the difference was not significant ( $89 \%$ vs. $76 \%, P=0.10$ ) after adjusting for other demographic variables.

\section{Support for development of the GMP under different enrollment models}

Three-quarters of participants believed the GMP should be developed if an opt-out approach were used, compared with $82 \%$ if an opt-in approach were used $(P=0.009$; Table 2$)$. High 
Table 2 Comparison of attitudes toward the use of opt-out and opt-in models of enrollment in the VA Genomic Medicine Program

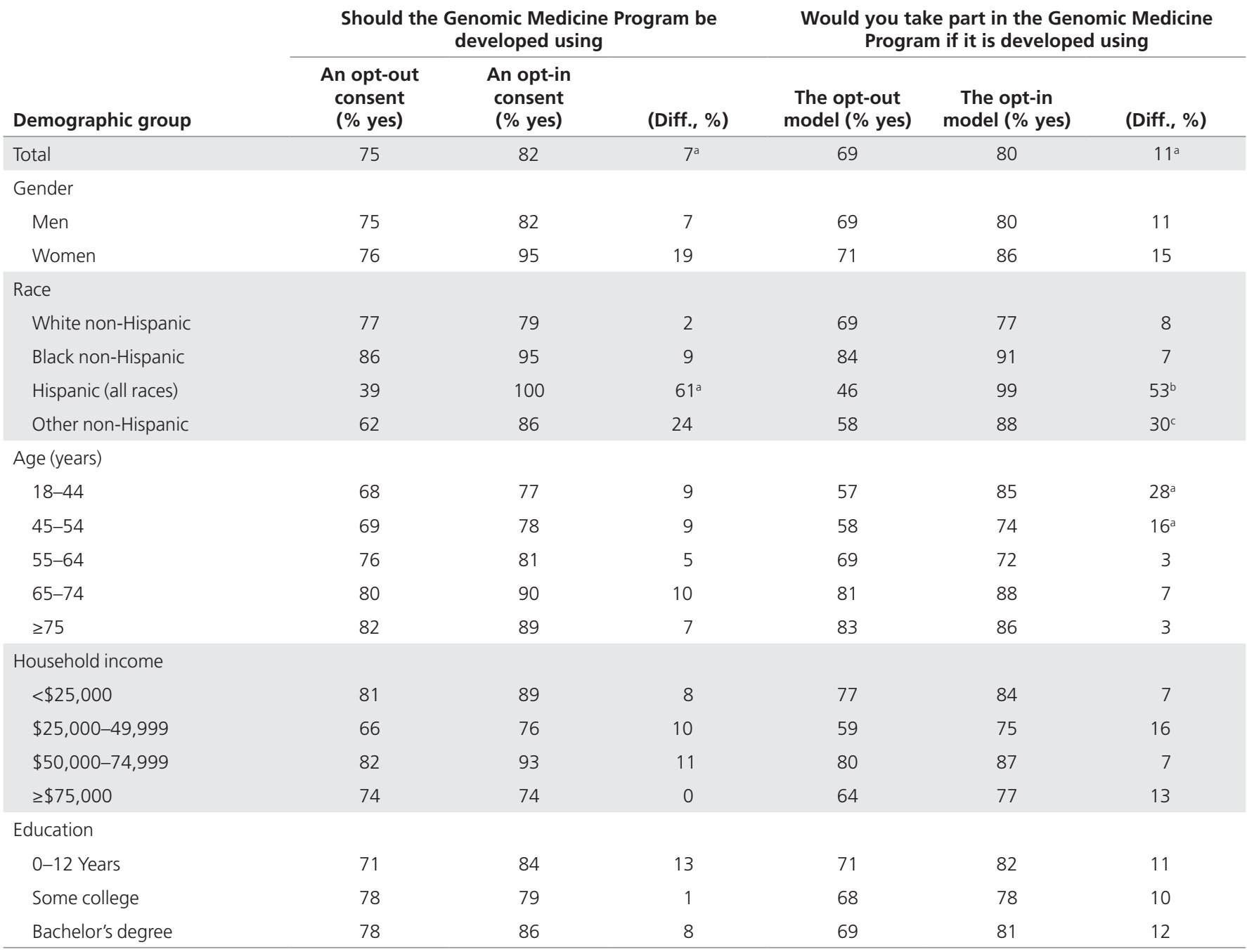

Diff., diffference; VA, Department of Veterans Affairs.

aDifference between support for/willingness to take part in opt-out consent and opt-in consent is statistically significant $(P<0.05)$ after Bonferroni correction for multiple comparisons. b Difference in willingness to participate under opt-out consent and opt-in consent is statistically significant $(P<0.0001)$ after Bonferroni correction for multiple comparisons. 'Difference in willingness to participate under opt-out consent and opt-in consent is not significant $(P<0.06)$ after Bonferroni correction for multiple comparisons.

levels of support ( $\geq 74 \%)$ for the use of opt-in recruitment and consent were observed in all demographic groups (Table 2). With the exception of Hispanics, majorities of all groups also supported developing the database using an opt-out approach. However, support for opt-out was lower than support for opt-in within every demographic group except people whose annual household income was $\geq \$ 75,000$.

Groups whose support for research was influenced most by the model of enrollment used included Hispanics, non-Hispanics of races other than black or white, and women; however, only the large difference observed within Hispanics was statistically significant (100\% supported opt-in; 39\% supported opt-out; $P=0.003$ ). White non-Hispanics and people with household incomes $\geq \$ 75,000$ showed the smallest differences in support for the models.
Overall, nearly two-thirds of respondents said they would support research using either model. A total of $17 \%$ would support the GMP only if opt-in were used; $10 \%$ would support it only if opt-out were used; and $8 \%$ would not support the program under either model.

\section{Beliefs and preferences about consent}

When asked how they would "prefer to be approached and consented to participate in the GMP," $29 \%$ of respondents chose the opt-in method, $14 \%$ chose the opt-out method, $50 \%$ said either would be acceptable, and 7\% said they would not want to be asked to participate. Responses to this question varied by demographic group (Figure 1). Hispanics, black non-Hispanics, and respondents who had completed at least a Bachelor's degree were more likely to say they would prefer an opt-in 


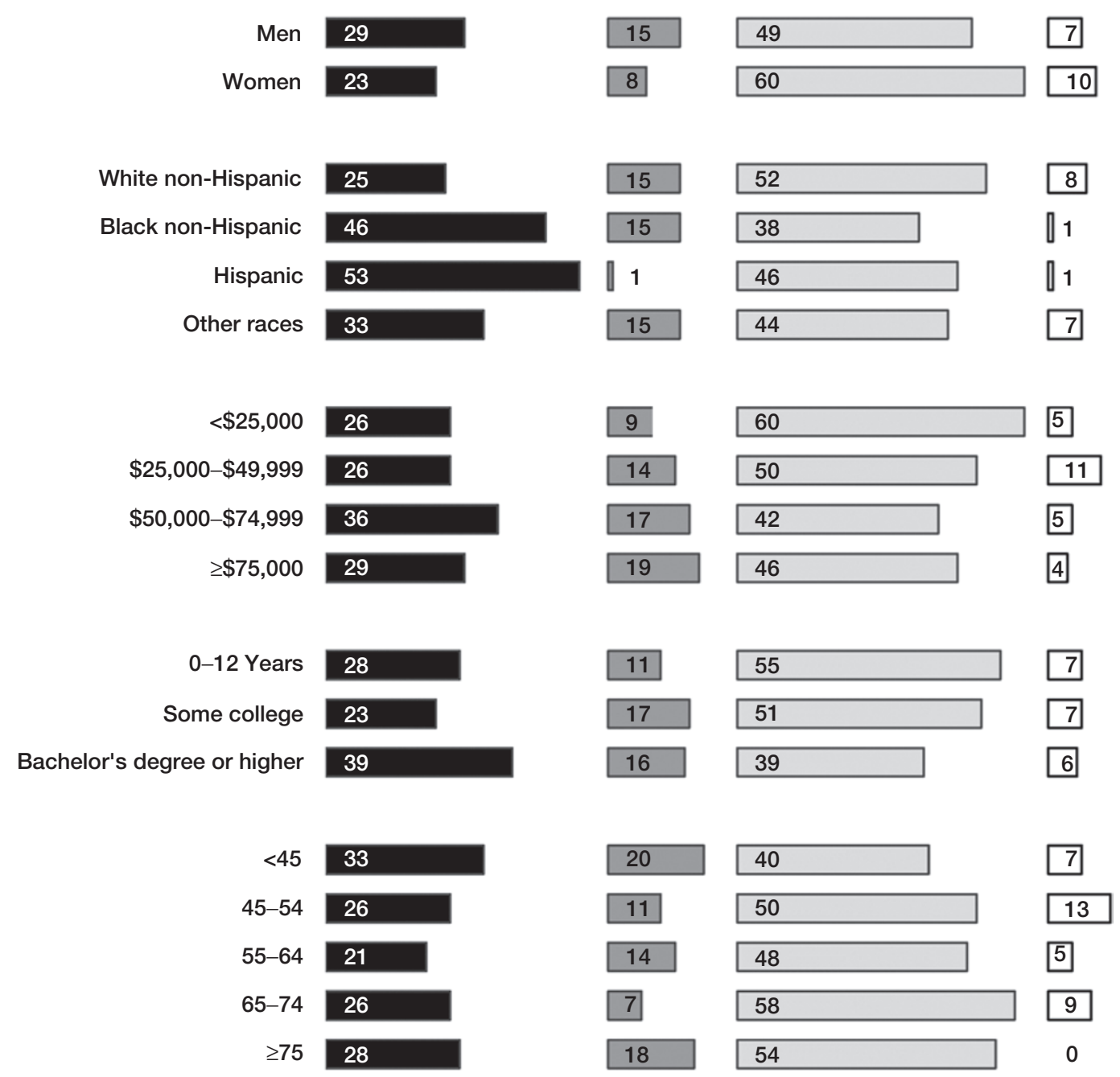

Figure 1 Preferences of VA patients $(n=451)$, by demographic group, about the enrollment method used to approach veterans about participating in the VA Genomic Medicine Program. Some rows do not total 100\% due to rounding errors. VA, Department of Veterans Affairs.

model. Respondents with household incomes $\geq \$ 75,000$ and those under 45 years of age were the most likely to prefer an opt-out model.

Veterans were also asked about several aspects of opt-in and opt-out enrollment. Similar numbers of people agreed that each method would make it easy to participate (Table 3). Slightly more people agreed that the opt-out model would be successful in recruiting participants from all segments of the VA, compared with the opt-in model ( $81 \%$ vs. $76 \%$ ). On the other hand, more people agreed the opt-in model would respect people's right to choose whether to participate (90\%), as compared with the opt-out model (86\%); and considerably more people believed the opt-out model would wind up including people in the database who did not really want to participate ( $47 \%$ vs. 27\%). Among those who agreed that opt-out enrollment would inadvertently include some unwilling veterans, those who still supported the use of opt-out methods $(n=167)$ were twice as likely as those who did not $(n=45)$ to agree that opt-out enrollment would make it easy to participate ( $94 \%$ vs. $49 \%$, respectively) and would gather participants from all segments of the VA ( $93 \%$ vs. $42 \%)$.

When asked about the opt-in model, 39\% preferred to give consent once for all the research that the MVP would undertake; $8 \%$ wanted to be asked only once but would want to be allowed to consent to specific categories of research; and 50\% preferred to be asked for consent for each study the GMP would conduct. Despite these preferences, if an opt-out approach were used, more than three-quarters would be very $(46 \%)$ or somewhat (31\%) comfortable with the fact that samples and information in the database could be used for any studies deemed acceptable by GMP researchers; $11 \%$ would be somewhat uncomfortable, and $13 \%$ very uncomfortable.

Participants were also asked about the acceptability of different methods of opting out. Nearly all respondents said signing 
Table 3 Veterans' opinions about various aspects of the enrollment process for the GMP, as they relate to both opt-in and opt-out methods of enrollment

\begin{tabular}{lcc}
$\begin{array}{l}\text { "In thinking about an (opt-in/opt-out) } \\
\text { approach to develop the GMP, do you } \\
\text { agree or disagree with the following } \\
\text { statements?" }\end{array}$ & $\begin{array}{c}\text { Opt-in } \\
\text { (\% who } \\
\text { agree) }\end{array}$ & $\begin{array}{c}\text { Opt-out } \\
\text { (\% who } \\
\text { agree) }\end{array}$ \\
\hline $\begin{array}{l}\text { "The method would make it easy to } \\
\text { participate" }\end{array}$ & 87 & 86 \\
$\begin{array}{l}\text { "The method would respect people's right } \\
\text { to choose whether to participate" }\end{array}$ & 90 & 86 \\
$\begin{array}{l}\text { "It is a good way to develop the database" } \\
\text { "It would get participants from all segments } \\
\text { of the VA" }\end{array}$ & 83 & 81 \\
$\begin{array}{l}\text { "It would include people who did not really } \\
\text { want to participate" }\end{array}$ & 27 & 81 \\
\hline "It would make me feel like a guinea pig" & 20 & 47 \\
\hline
\end{tabular}

GMP, Genomic Medicine Program; VA, Department of Veterans Affairs.

a form at the time of a surgery or blood draw at the VA (96\%) or during a visit to their VA physician $(90 \%)$ would be acceptable ways to opt out. Fewer people said that opting out by mail (65\%), calling the VA (51\%), completing an online form (45\%), or e-mailing the VA (43\%) were acceptable. A total of $51 \%$ would want to be given the opportunity to opt out each time they give blood or undergo surgery at the VA, whereas $28 \%$ would prefer to be asked periodically-either at the VA or via a reminder sent home-and $21 \%$ would want to be asked only once.

If an opt-in method were used, $9 \%$ would prefer to have blood drawn specifically for the database, $24 \%$ would prefer that the study use "leftover blood or tissue from my medical care," and $54 \%$ said that either method would be acceptable. The remainder (12\%) said they would not participate.

\section{Willingness to participate in the GMP under different models of enrollment}

Respondents were asked whether they would participate in the GMP if the study used the opt-in model and the opt-out model, randomizing the order of the two questions. Overall, $80 \%$ said they would definitely $(36 \%)$ or probably $(44 \%)$ participate if opt-in were used, and $69 \%$ would be willing to participate (31\% definitely and $38 \%$ probably) if opt-out were used ( $P=$ 0.0001 ; Table 22. Nearly two-thirds of respondents (64\%) said they would take part in both opt-in and opt-out versions of the GMP; $16 \%$ would only take part if opt-in were used, $5 \%$ would participate only if opt-out were used, and $14 \%$ would not participate in either case.

In all demographic groups, at least $70 \%$ of respondents would be willing to participate in the research program if they were allowed to opt in Table 2. Willingness to participate in a study using the opt-out approach was lower in every demographic group. The enrollment model used was significantly related to willingness to participate among Hispanics $(P=0.00007)$ and people under the age of $55(P=0.00001)$.
Of the opinions listed in Table 3, the belief that the opt-in approach "respects people's right to choose whether to participate" was the only significant predictor of willingness to take part in an opt-in study ( $86 \%$ vs. $30 \%, P=0.002$, adjusting for age, gender, race and ethnic background, education, and income). This relationship was not observed for the opt-out model. Instead, the belief that an opt-out approach would make it easy to participate was significantly related to willingness to take part in an opt-out study ( $77 \%$ vs. $36 \%$, adjusted $P=0.04$ ).

\section{Comparisons with the 2008 VA survey population}

To ascertain whether excluding members of the KN panel who participated in the 2008 survey biased the current sample, several questions from the 2008 survey were repeated and compared with responses from the 2008 sample. No statistically significant differences were noted in the overall responses, after adjusting for demographic factors.

A notable difference between responses to the 2008 and 2009 survey was observed among black non-Hispanic participants. Willingness among black non-Hispanics to participate in the GMP-regardless of which model was being discussed-was higher in the 2009 survey than in the 2008 survey (Figure 2); $91 \%$ said they would take part in an opt-in study (odds ratio adjusted for age, gender, income and education $=9.5, P=0.004$ ), and $84 \%$ would take part in an opt-out study (adjusted odds ratio $=2.0, P=0.12$ ), as compared with $51 \%$ of black non-Hispanic participants, who said they would take part in the study with an unspecified enrollment model in 2008. In comparison, in other races and ethnic groups, willingness to participate in an opt-out study in 2009 was lower than willingness expressed in $2008 .{ }^{9}$

\section{DISCUSSION}

Support for the GMP and willingness to participate in the database was high among veterans. Half of survey respondents showed no preference for opt-in or opt-out methods of enrollment. The observation that significantly more respondents would be willing to take part in a study using traditional opt-in consent is consistent with a meta-analysis of enrollment preferences for use of previously collected samples, which found that most people want an opportunity to actively decide to participate. $^{13}$

The observation that among those with a preference, roughly twice as many veterans preferred the opt-in model has been echoed elsewhere. ${ }^{14,15}$ Assuming that the percentages of respondents who said they would participate under the respective models actually applied to the MVP study, recruiting 1,000,000 subjects would require asking 1,450,000 veterans using an optout method or 1,250,000 veterans using an opt-in method. In practice, however, the opt-out rate of one large cohort study has been reported to be $<5 \%$, and, in a small, randomized-controlled trial of opt-in and opt-out enrollment models, participants in the opt-out group were more satisfied than those who were asked to opt in. ${ }^{16}$

Perhaps more important than the absolute numbers needed to reach recruitment goals under the two models is the finding that 


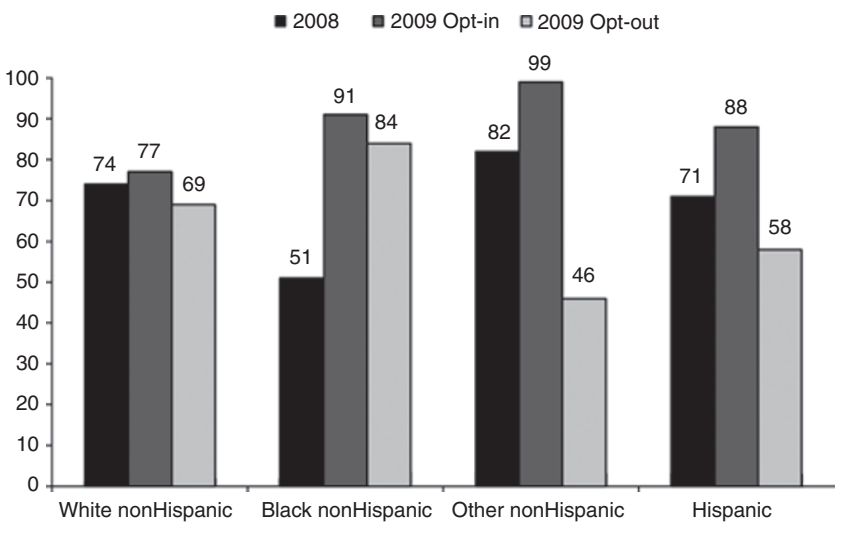

Figure 2 Percent of surveyed VA patients $(n=451)$, by race/ethnic group, who would be willing to paricipate in the Genomic Medicine Program. Results of 2008 and 2009 surveys. VA, Department of Veterans Affairs.

support for and willingness to participate in an opt-in study are consistent across demographic groups. In contrast, the data suggest that using an opt-out model may make it more difficult to recruit representative samples of Hispanics and veterans under the age of 55 years (Table 2). In addition, in the VA health-care system, which includes hundreds of care centers of various sizes, patient populations, and research resources, there may be variability in whether and how VA staff provide patients with opportunities to opt out. Use of the opt-out method could, therefore, result in the inclusion of more participants who did not intend to enroll than would occur in a localized health-care system.

One potential advantage of an opt-out model is that collection of additional blood or tissue samples would be unnecessary. Veterans' opinions suggest, however, that this benefit may also be realized under an opt-in model in the VA system. One in four respondents said that in an opt-in study, they would prefer that a residual clinical sample be used, and 54\% stated no preference between using a residual sample and collecting a new sample. These data suggest that the appeal of the opt-in model is not strongly related to a desire for control over blood and tissue samples, and is more likely related to the importance of being explicitly asked to participate. This is supported by the strong relationship between stated willingness to participate and the belief that opt-in consent respects people's right to choose whether to take part-and the absence of this relationship when people consider the opt-out model. ${ }^{9}$

Of the $47 \%$ of respondents who agreed that opt-out methods would enroll people who did not want to participate, $78 \%$ also agreed that using opt-out was a "good way" to enroll people. This does not necessarily mean that moral issues were not considered when evaluating whether the opt-out method was a "good" one. We believe, rather, that people weighed the possible practical advantages of opt-out enrollment against the potential moral hazards associated with inadvertently including unwilling VA patients, and, on average, the practical advantages carried more weight. A different belief, respecting people's right to choose whether to participate, was related to support for the opt-in model.
Another interesting observation is that willingness among black non-Hispanics to participate in an opt-in study was significantly higher than willingness to participate among black non-Hispanics surveyed in 2008. ${ }^{9}$ Studies have found that black non-Hispanics may be more likely to distrust the biomedical research community and, as a result, might be more positively influenced by increased transparency about methods for study enrollment and sample collection. However, it is also possible that the observed difference is attributable to other factors or temporal effects we did not measure.

One limitation of this study is the relatively small size of the survey, which limits our ability to make meaningful comparisons of attitudes between some demographic subgroups, such as between men and women or among non-Hispanics of races other than white or black. Another possible limitation of the study may stem from the descriptions of opt-out enrollment used in the survey. The intent was to describe a realistic, relatively passive process by which the VA would notify veterans about the study and the requirements for opting out (see Supplementary Data online). However, allusions to a form to be signed in order to opt out, and to possible methods the VA would use to collect opt-out preferences could have led survey respondents to perceive the model as a more active, solicitous process on the part of the VA. This interpretation may have increased support for the opt-out method.

Another important limitation is that the survey asks about veterans' preferences and willingness to participate in what was, at the time, a hypothetical study. Although many VA patients have had prior experience taking part in biomedical research studies at the VA, people's responses on a survey about their willingness to take part in a hypothetical study will not always correlate with actual behaviors. An additional limitation is one that is common to most studies on the conduct of research: survey participants have agreed to take part in this study and are members of the $\mathrm{KN}$ panel; they may thus be more favorably inclined toward biomedical research than the general VA population. This study is likely to provide valid estimates of the relative levels of support for the GMP under opt-in and opt-out scenarios but may overestimate actual support and participation rates.

The VA began recruiting for the MVP in January 2011, using an opt-in model to enroll participants. As the MVP moves forward, additional research could assess participation rates, how these rates vary among demographic and other groups, and what study design factors might be changed or enhanced to improve recruitment and retention of participants. Furthermore, the data presented here may be of use to other biobanks and research programs weighing the relative acceptability of opt-in and opt-out designs.

\section{DISCLOSURE}

The authors declare no conflict of interest.

\section{SUPPLEMENTARY MATERIAL}

Supplementary material is linked to the online version of the paper at http://www.nature.com/gim 


\section{REFERENCES}

1. Manolio TA. Cohort studies and the genetics of complex disease. Nat Genet 2009;41:5-6.

2. Collins FS, Manolio TA. Merging and emerging cohorts: necessary but not sufficient. Nature 2007;445:259.

3. McCarty CA, Wilke RA, Giampietro PF, Wesbrook SD, Caldwell C. Marshfield Clinic Personalized Medicine Research Project (PMRP): design, methods and recruitment for a large population-based biobank. Personalized Med 2005;2:49

4. National Children's Study, 2012. http://www.nationalchildrensstudy.gov Accessed 11 January 2012.

5. Kaiser Permanente Division of Research, 2011. http://www.dor.kaiser.org Accessed 11 January 2012.

6. Roden DM, Pulley JM, Basford MA, et al. Development of a large-scale de-identified DNA biobank to enable personalized medicine. Clin Pharmacol Ther 2008;84:362-369.

7. Department of Veterans Affairs. Veterans Health Administration, 2011. http://www.va.gov/health/aboutVHA.asp. Accessed 6 December 2011.

8. US Department of Veterans Affairs. Million Veteran Program (MVP), 2011 http://www.research.va.gov/mvp/. Accessed 11 January 2012.
9. Kaufman D, Murphy J, Erby L, Hudson K, Scott J. Veterans' attitudes regarding a database for genomic research. Genet Med 2009;11:329-337.

10. SPSS for Windows. Version 19. SPSS: Chicago, IL, 2011

11. RTI International. SUDAAN, Version 9. RTI International: Research Triangle Park, NC, 2011.

12. Department of Veterans Affairs Office of the Assistant Deputy Under Secretary for Health Policy and Planning. 2005 Survey of Veteran Enrollees' Health and Reliance Upon VA. http://www.va.gov/healthpolicyplanning/ reports/FinalSOE_05.pdf. Accessed 11 January 2012.

13. Wendler $D$. One-time general consent for research on biological samples. BMJ 2006:332:544-547.

14. Vermeulen E, Schmidt MK, Aaronson NK, Kuenen M, van Leeuwen FE. Obtaining 'fresh' consent for genetic research with biological samples archived 10 years ago. Eur J Cancer 2009;45:1168-1174.

15. Willison DJ, Schwartz L, Abelson J, et al. Alternatives to projectspecific consent for access to personal information for health research: what is the opinion of the Canadian public? J Am Med Inform Assoc 2007; $14: 706-712$

16. Junghans $C$, Feder $G$, Hemingway $H$, Timmis A, Jones $M$. Recruiting patients to medical research: double blind randomised trial of "opt-in" versus "optout" strategies. BMJ 2005;331:940. 